\title{
Reconciling measured scattering response of 3D metamaterials with simulation
}

\author{
Bryan M. Adomanis ${ }^{1}$, Paul J. Resnick ${ }^{2}$, and D. Bruce Burckel ${ }^{2, *}$ \\ 1 Air Force Research Laboratory, 3005 Hobson Way, Wright-Patterson AFB, OH 45433, USA \\ 2 Sandia National Laboratories, P.O. Box 5800, Albuquerque, NM 87185, USA
}

Received 31 August 2015 / Accepted 17 November 2015

\begin{abstract}
Membrane projection lithography is used to create 3-dimensional unit cells in a silicon matrix decorated with metallic inclusions. The structures show pronounced resonances in the 4-16 $\mu \mathrm{m}$ wavelength range and demonstrate direct coupling to the magnetic field of a normally incident transverse electromagnetic (TEM) wave, a behavior only possible for vertically oriented resonators. Qualitative agreement between rigorous coupled wave analysis (RCWA) simulation and measured scattering response is shown. COMSOL simulations show that slight variations in both metallic inclusion and silicon unit cell physical dimensions can have large impact in the scattering response, so that design for manufacture of 3D metamaterial structures for applications should be done with care.
\end{abstract}

Key words: 3D Metamaterials, Split Ring Resonator, Micro Fabrication.

\section{Introduction}

Structured electromagnetic materials form the basis for many recent exciting developments in electromagnetic research. From photonic crystals [1], to metamaterials [2] and plasmonics [3], the ability to fabricate and structure materials at sub-wavelength scales has consistently enabled new avenues for control over the interaction between electromagnetic energy and materials. Recently, techniques such as direct laser write [4, 5], nano-origami [6, 7], layer-by-layer e-beam lithography $[8,9]$ and interferometric lithography $[10,11]$ have all been used to create complex $3 \mathrm{D}$ structures at the micrometer scale. Despite this interest and research effort, fabrication of subwavelength micron-scale 3-dimensional structures for infrared and optical wavelength applications continues to present a formidable challenge. Due to the practical constraints of 3-dimensional fabrication at the micro and nano scale, structured electromagnetic materials research is typically lead by theory, where the scattering behavior of materials composed of arrays of complex unit cells can be studied in the domain of numerical simulation. As the field of 3D micro/nanofabrication matures to include fabrication techniques such as direct laser write, nano-origami and layer-by-layer fabrication, structures resembling the numerical models can be fabricated and tested to confirm or contradict the predictions of the simulations.

One emerging 3D-fabrication approach is membrane projection lithography (MPL) [12, 13]. Membrane projection

*e-mail: dbburck@sandia.gov lithography is capable of fabricating large area dense arrays of micrometer-scale unit cells. Originally demonstrated as a "boutique" fabrication approach using polymer materials and e-beam written patterns, MPL has recently been transitioned into a complementary metal oxide semiconductor (CMOS) compatible version using inorganic materials and standard CMOS processing equipment [14]. In addition to enabling fabrication of micrometer-scale $3 \mathrm{D}$ metallic structures, translation of MPL into inorganic materials, such as silicon, removes many of the organic resonances present in polymer matrices which cloud the interpretation of structural resonances of the metallic inclusions.

In this paper we review the MPL fabrication approach, and present optical characterization of a variety of $3 \mathrm{D}$ metallic inclusions created using MPL. The measured optical response of a split ring resonator (SRR) structure, perhaps the most studied metamaterial inclusion, is then compared with numerical simulations. We find that only qualitative agreement between measured and modeled data is achieved. Without rigorous agreement between measured and modeled data, assigning physical mechanisms for the observed resonances based on numerical simulation will not be accurate. In an attempt to reconcile the difference between measured and modeled performance, we perform sensitivity analysis on a split-ring resonator structure and demonstrate that minor structural differences in the 3D SRR geometry specific to the MPL fabrication approach can lead to large changes in optical scattering response. 


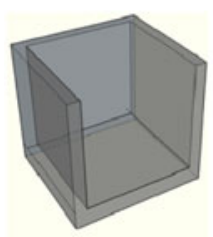

(a)

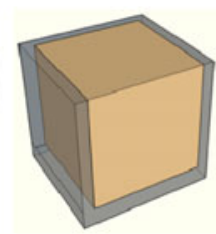

(b)

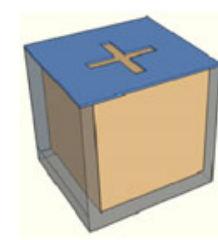

(c)

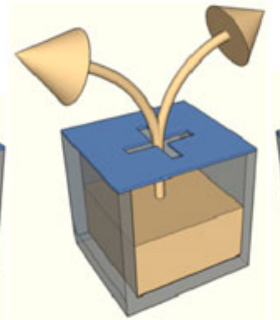

(d)

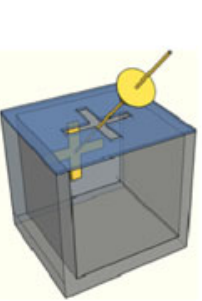

(e)

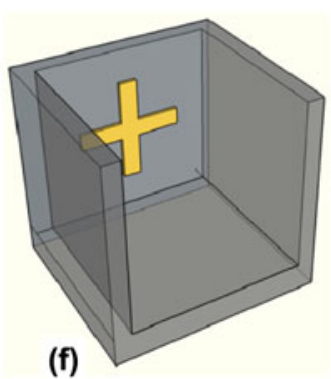

Figure 1. Schematic sequence of CMOS-compatible membrane projection lithography process.

\section{Fabrication}

Figure 1 contains a schematic depiction of the fabrication process for a single unit cell of a larger array. A unit cell cavity is etched into silicon (Figure 1a), and backfilled $\mathrm{SiO}_{2}$ and chemically mechanically polished flat (Figure $1 \mathrm{~b}$ ). An aluminum nitride (AlN) layer is deposited and patterned using a standard $248 \mathrm{~nm}$ ArF stepper (Figure 1c). In this case, a cross is shown, but the pattern can be any self-supporting structure. Hydrofluoric acid is then used to evacuate out the $\mathrm{SiO}_{2}$ from the unit cell cavity through the patterned AlN membrane, yielding a suspended, patterned membrane positioned over the unit cell (Figure 1d). Angled, directional deposition places a replica of the membrane pattern on an interior face of the unit cell (Figure 1e), and a high $\mathrm{pH}$ aqueous liftoff removes the metal-coated AIN membrane, yielding the final structure (Figure 1f).

Figure 2 contains SEM images of (a) a split ring resonator (SRR) and (b) a gammadion fabricated using MPL processing. The samples were fabricated on $150 \mathrm{~mm}$ silicon wafers in Sandia's CMOS fab. As drawn on the mask, the cavities are nominally $2 \mu \mathrm{m}$ wide and the walls are nominally $300 \mathrm{~nm}$ thick. The as drawn linewidth of the inclusions is $350 \mathrm{~nm}$, and the inclusion is formed from a $\mathrm{Cr} / \mathrm{Au}$ stack with thickness of $10 \mathrm{~nm} / 50 \mathrm{~nm}$. Although multiple evaporations could be performed to place multiple instances of each pattern in the unit cell, in this case, just a single instance was evaporated at $45^{\circ}$ relative to the surface normal, depositing a single inclusion on the wall shown. The rounding of the corners for the gammadion and the incomplete formation of the horizontal "arms" of the structure were due to a slight protruding veil formed during AlN patterning (not shown) which shadowed the pattern clear aperture during the angled metal deposition. This etch problem has since been resolved. Additionally, the $\mathrm{SiO}_{2}$ sacrificial backfill was incompletely evacuated in both samples. Although the material contrast is not apparent in these SEM images, the small voids indicated by the arrows in Figure $2 b$ show the true bottom extent of the unit cell (indicated by the dotted lines). The material between these voids and the "false" bottom of the unit cell is un-removed $\mathrm{SiO}_{2}$. Both samples possess this oxide plug, which can be eliminated with a longer $\mathrm{HF}$ evacuation.

One advantage of silicon is that it is transparent in the infrared portion of the spectrum. Although it is transparent it also has a large index of refraction ( 3.4), so that the silicon, both in the walls of the unit cells as well as the substrate, where

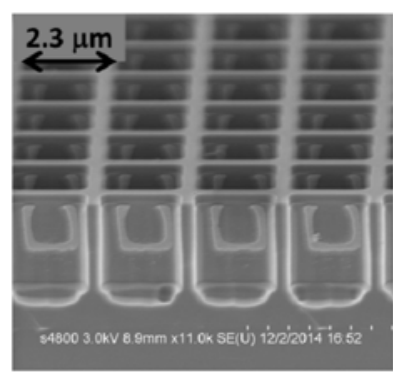

(a)

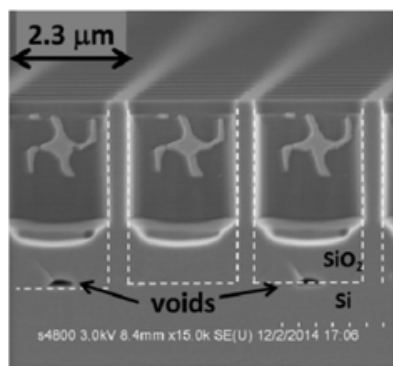

(b)
Figure 2. (a) SEM image of a 2D array of unit cells with vertically oriented split ring resonator inclusions; (b) SEM image of a 2D array of unit cells with vertically oriented gammadion inclusions.

multiple diffraction orders and multiple internal reflections can occur, profoundly affect incident light. In order to minimize this effect, the fabricated structures were interrogated in reflection, rather than in transmission. The reflection spectra of the samples were measured using a Nicolet 6700 FTIR at $10^{\circ}$ relative to the sample normal using an SOC-100 hemispherical directional reflectometer (HDR). The data is shown in the graphs of Figure 3 for both the split ring resonator (Figure 2a) and the gammadion samples (Figure $2 b$ ) for linear polarization with the E-field oriented parallel to the vertical plane of the inclusions, and perpendicular to the plane of the inclusions. The signals are normalized to a gold mirror for reference, representing $100 \%$ reflectivity on the graphs, but otherwise the data is presented in its raw form with no data smoothing or other post-processing. The dots in the graphs represent distinct measurements. For the perpendicular polarization, neither the electric field nor the magnetic field is oriented such that they can drive current oscillations in the inclusions. For the parallel polarization, the magnetic field is oriented perpendicular to the plane of the SRR, and the resulting flux can magnetically induce current flow in the fundamental mode of the SRR. Additionally, the electric field can excite both the magnetic resonance of the SRR as well as higher order electric resonances in both the SRR and gammadion samples.

For the perpendicular polarization, the reflectivity has moderate amount of modulation in both samples owing to the high index contrast of the silicon/air interface and the high aspect ratio modulation of the silicon surface. Additionally, both samples show an obvious material absorption peak at $\sim 9 \mu \mathrm{m}$ due to the presence of the un-removed $\mathrm{SiO}_{2}$ in the 


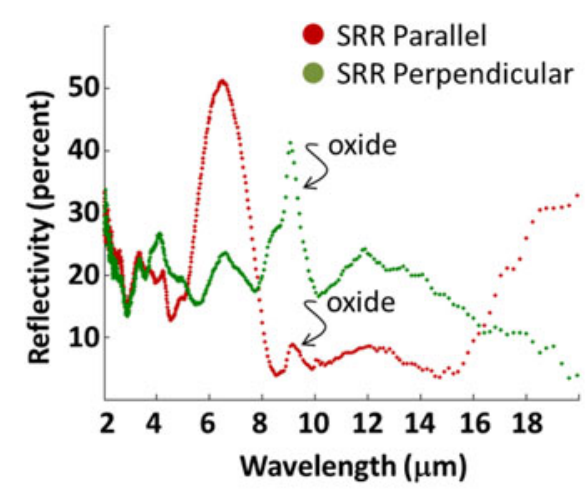

(a)

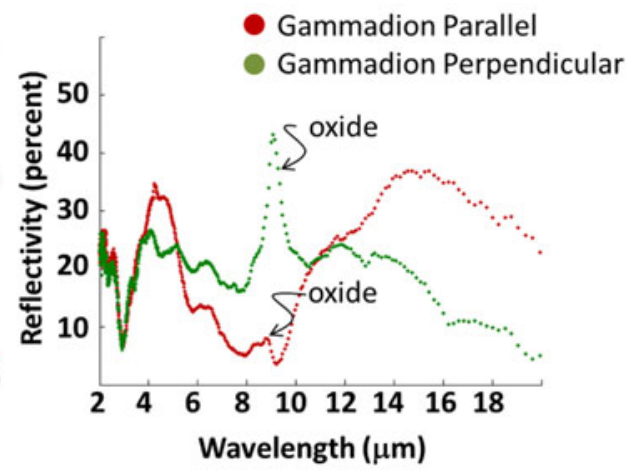

(b)

Figure 3. Measured reflectivity for (a) the SRR and (b) the gammadion samples of Figure 2.

bottoms of the unit cells. For the parallel polarization, the SRR sample shows a pronounced, symmetric resonance peak at $\sim 6.5 \mu \mathrm{m}$, while the gammadion sample shows a resonance peak at $\sim 4.5 \mu \mathrm{m}$. In both samples, the oxide absorption peak is dramatically reduced in the parallel polarization even though neither inclusion possesses a structural resonance at this spectral location.

\section{Simulation}

In $[15,16]$ parametric studies of microwave regime metamaterials were performed, showing how variations in SRR dimensions impact the scattering behavior of the structures. The metamaterial inclusions created using MPL in this work possess structural features specific to the fabrication approach, which impact the scattering response in a way which is not captured in previous sensitivity studies. In this work we used both rigorous coupled wave analysis (RCWA), a frequency-based approach, as well as COMSOL, a finite element approach. Dispersive optical constants for both silicon and gold were measured using a J.A. Woollam infrared variable angle spectroscopic ellipsometer (IR-VASE). These optical constants were used for all simulations in this work.

Figure 4a contains an SEM image of SRR structures fabricated without the residual oxide in the bottom of the unit cell, and without the AIN etch veils which caused the previous SRRs to suffer from excessive linewidth variation. During fabrication a keyhole developed in the oxide backfill resulting in the inclusion of a coaxial element in the center of the SRR [14]. This coaxial element has only a small effect on the optical response of the SRR, but has been included in all the simulations performed. The MPL process can be adjusted to remove this feature.

Figure $4 \mathrm{~b}$ contains schematic diagrams of the unit cell and inclusions with the relevant physical dimensions identified. Figure 5 contains graphs of the normalized measured and RCWA-modeled scattering response for the coaxial SRR structure for two separate orientations of the SRR with respect to the wafer surface compared to the measured signal.

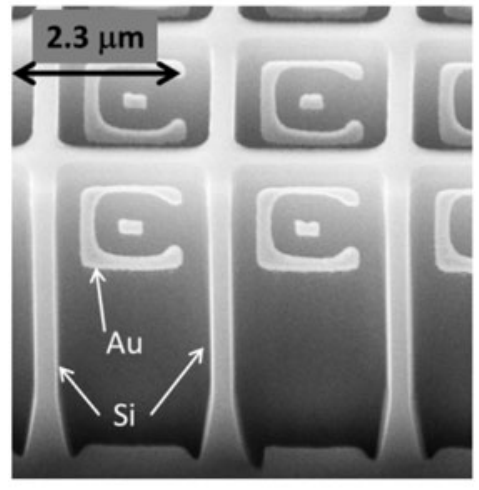

(a)

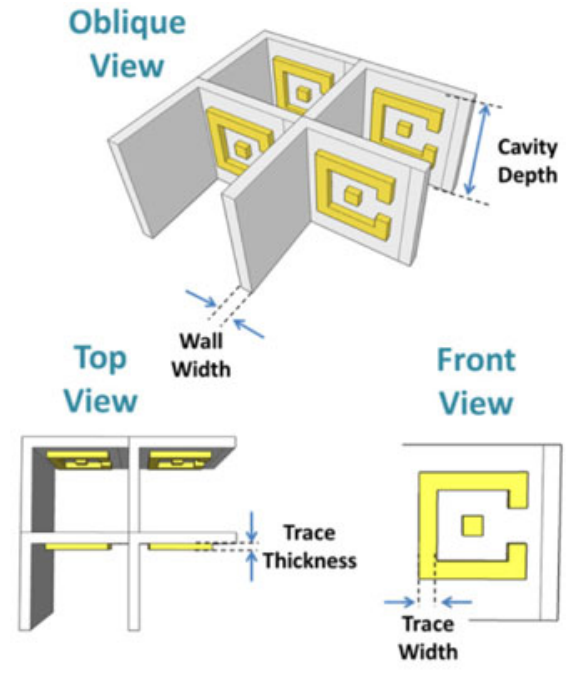

(b)

Figure 4. (a) SEM image of coaxial SRR array fabricated and measured in Figure 5; (b) schematic diagrams showing the inclusion and unit cell dimensions which are varied in the sensitivity analysis below. 


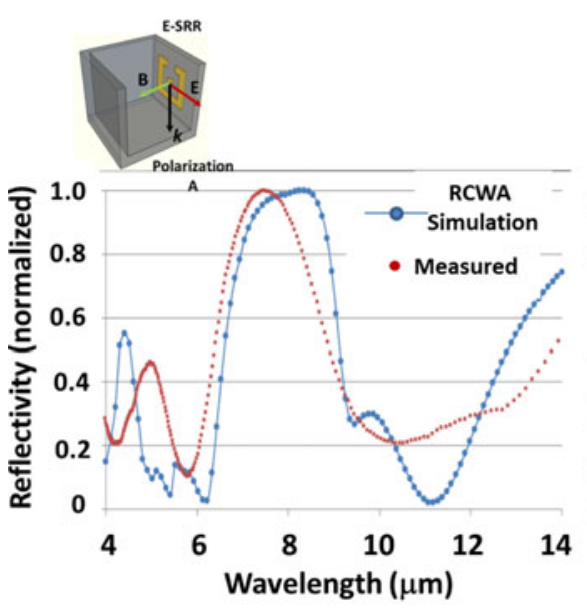

(a)

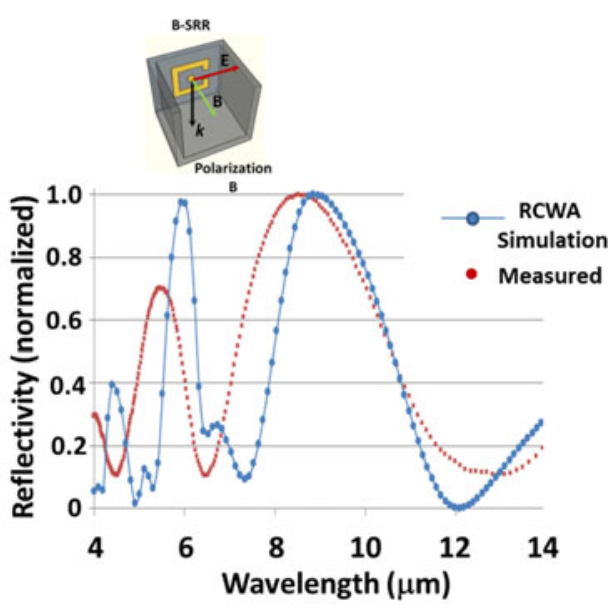

(b)

Figure 5. Measured and RCWA simulated reflectivity for (a) an electrically excitable SRR with gap oriented parallel to the wafer surface; (b) a magnetically excitable SRR with gap oriented perpendicular to the wafer surface. The data markers represent measured/modeled data points. The data has been normalized to unity by dividing every point by the maximum value, but is otherwise un-altered.

In Figure 5a, SRR gap is oriented parallel to the surface, while in Figure 5b, the gap is oriented vertical with respect to the wafer surface. For both orientations, only the active polarization (noted in the inset schematics) is shown. Both orientations demonstrate a fundamental magnetic resonance at $\sim 8 \mu \mathrm{m}$, with higher order resonances at shorter wavelengths. For the orientation in Figure 5a both the magnetic and electric fields can excite the fundamental magnetic resonance. For the orientation in Figure 5b, the magnetic resonance can only be excited by the magnetic field, so the resonant effect is unambiguously magnetic in nature. The measured and modeled data were both normalized to unity maxima for comparison, but the data is otherwise in its raw form with no averaging or smoothing. We attribute the smoothness of the data to the complete removal of the oxide in the unit cells as well as increased uniformity in the inclusions due to the removal of the AIN etch veils. The modeled retained the lowest 23 modes. The physical dimensions of the modeled structure were adjusted to provide a qualitative fit between the measured and modeled data (cavity width $=1.85 \mu \mathrm{m}, \quad$ depth $=3.75 \mu \mathrm{m}, \quad$ linewidth $=$ $300 \mathrm{~nm}$, SRR width $/$ length $=1400 \mathrm{~nm}$, gap $=950 \mathrm{~nm}$, coaxial width $=350 \mathrm{~nm}$ ). While qualitative agreement is achieved, the model possesses much more fine detail for both polarizations.

One possible explanation for the difference between measured and modeled data is that the physical dimensions of the structure are different than the modeled structure. The MPL fabrication approach possesses several possible contributions to uncertainty in the physical dimensions of the structure.

In order to study the impact of structural variations on the scattering response, COMSOL simulations were performed. Periodic boundary conditions were used at the lateral edges of the unit cell. An input port of appropriate polarization is located three unit cell heights over the top of the silicon walls. The reflection is monitored at this location as well. In Figure 6, the impact of variation of the angle of deposition on resonance wavelength was studied. Figure $6 \mathrm{a}$ shows the structures modeled for deposition angles ranging from $28^{\circ}$ to $45^{\circ}$, demonstrating elongation of the as-drawn SRR for deposition angles steeper than $45^{\circ}$. Resonance wavelength is shown to depend heavily on the angle of deposition, with the red shift in resonance peak correlated to the trace length of the SRR as expected. Because MPL is a purely geometric process, the impact of varying evaporation angle on the vertical dimensions of the SRR are given by $y_{\text {actual }}=\frac{y_{\text {nominal }}}{\tan (\theta)}$. It is somewhat surprising that variation of angle by $4^{\circ}$ as shown in Figure $5 \mathrm{~b}$ results in such a dramatic shift in the resonance.

Uncertainty in evaporation angle affects all unit cells equally. Some process specific variation could affect individual inclusions or unit cells. Depending on process maturity and tolerances for fabrication steps such as lithography and etch, it is quite likely that individual dimensions such as the wall width, inclusion linewidth and cavity depth could vary from edge to center of wafer, across an individual die, or in extreme cases, from unit cell to unit cell. In Figure 7, the effects of variation of some of these types of physical dimensions [(a) cavity depth, (b) trace width, (c) wall thickness and (d) trace thickness] are examined. In Figure $7 \mathrm{a}$, the cavity depth is varied by $500 \mathrm{~nm}$ for an SRR with gap width, $g=950 \mathrm{~nm}$, wall thickness, $t=300 \mathrm{~nm}$, trace width, $w=350 \mathrm{~nm}$, and SRR length $/$ width $=1500 \mathrm{~nm}$. Only modest changes are seen in the resonant response of the SRR. This is consistent with the measured data in Figure 3, where even off-resonance, incident light polarized parallel to the inclusion was relatively insensitive to the presence of the oxide in the bottom of the cavities. In Figure $7 \mathrm{~b}$, trace width is varied in $50 \mathrm{~nm}$ steps, shrinking the trace width symmetrically about the trace center line. In contrast to cavity depth variation, this form of dimensional variation has a large impact to the scattering response, impacting both the position and strength of several resonant features. In Figure $7 \mathrm{c}$, the wall thickness is varied in steps of $50 \mathrm{~nm}$. Again, this has a pronounced impact on the scattering response, resulting in a monotonic red shift in the resonant peaks. Finally, in Figure $7 \mathrm{~d}$, the thickness of the traces is varied; however this has a relatively minor impact on the scattering response. 

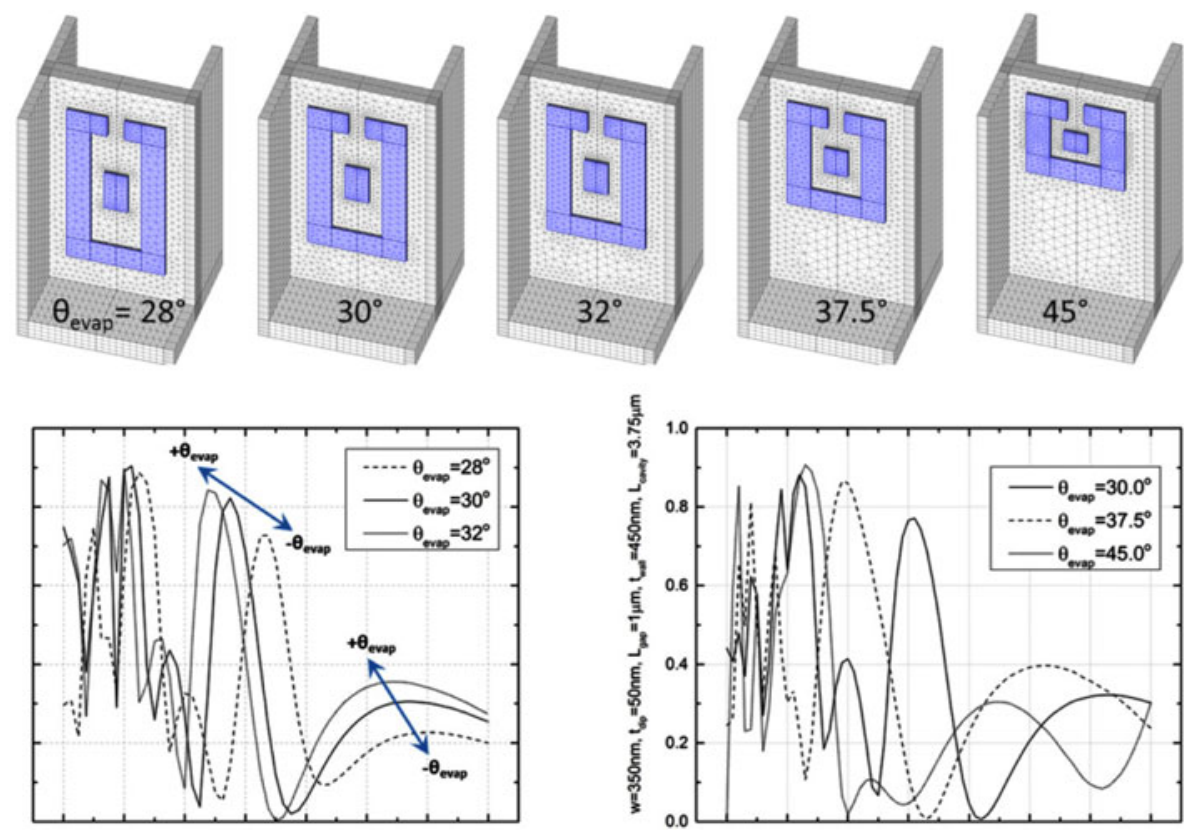

Figure 6. (a) Simulated structures where angle of evaporation was varied from $28^{\circ}$ to $45^{\circ}$; (b) scattering response for $28^{\circ}, 30^{\circ}$ and $32^{\circ}$; (c) scattering response for $30^{\circ}, 37.5^{\circ}$ and $45^{\circ}$.
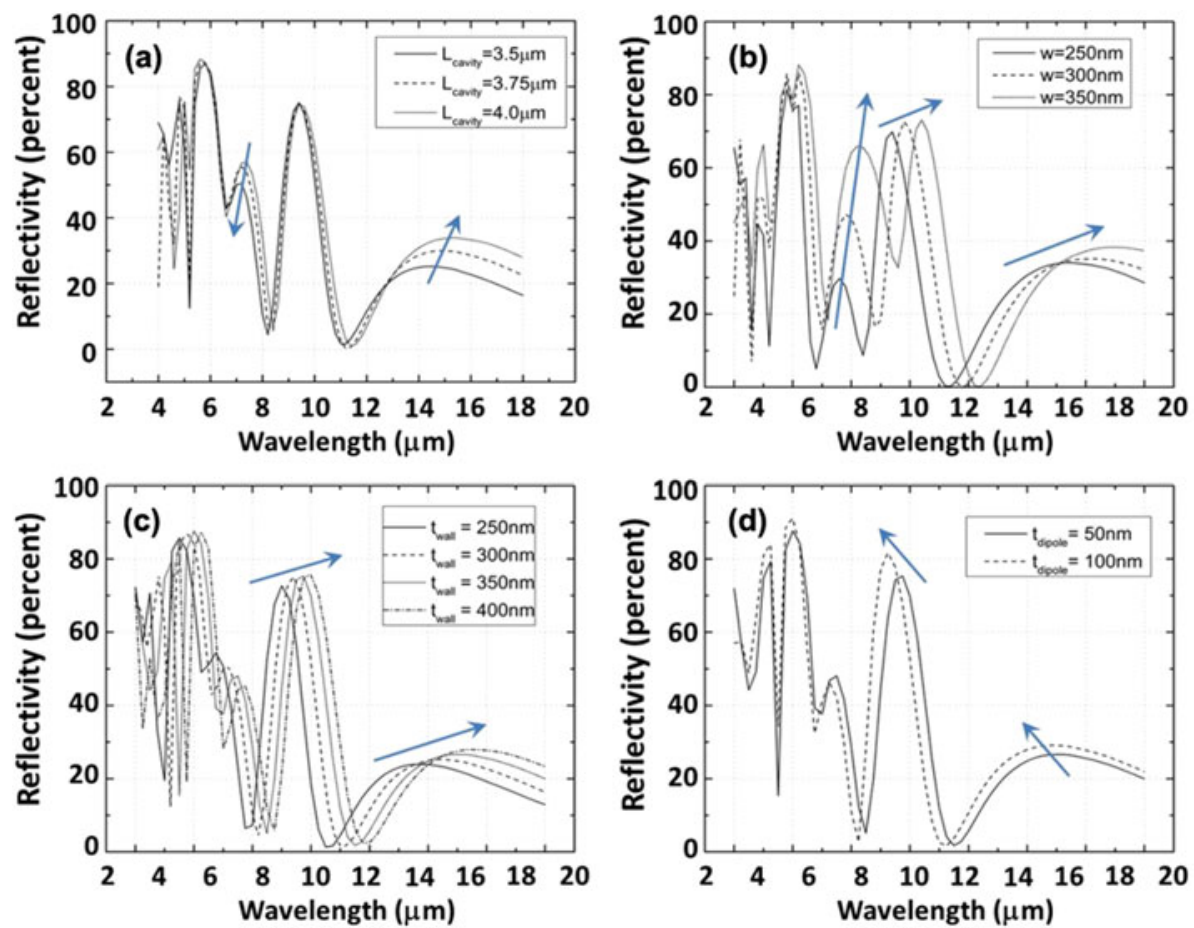

Figure 7. COMSOL simulations showing change in scattering response with (a) variation in cavity depth; (b) variation in trace linewidth; (c) variation in wall thickness; (d) variation in trace thickness.

\section{Implications and influences}

A CMOS compatible fabrication approach for creation of 3D metamaterial structures was outlined. Measured infrared spectra were obtained for two sample inclusion designs, exhibiting both structural resonances as well as suppression of material absorption resonances. RCWA simulations were shown to provide qualitative agreement with the scattering response for coaxial SRR structures. Using COMSOL, parametric sweeps of physical parameters reveal that for some physical SRR dimensions, a slight change in physical dimensions can lead to a disproportionately large change in the 
scattering response. This result has far reaching implications for fabrication and characterization. Depending on the fabrication tolerances of the manufacturing process, variations of physical parameters from wafer to wafer, across a single wafer or even a single die, could be $50 \mathrm{~nm}$ or more. The sensitivity analysis shown in Figures 6 and 7 demonstrate that variations of this degree can have a profound impact on the scattering behavior of the structure, making design for a particular scattering response challenging. While boutique fabrication approaches such as e-beam writing over small areas are inherently more immune to this variation, a technique such as MPL which can be used to create wafer-scale arrays of material is susceptible to dimensional shifts, or ensemble averages of structures rather than homogeneous arrays and hence changes in the electromagnetic behavior. Much like inhomogeneous broadening which affects the linewidth of quantum dot lasers, practical nano-photonic systems also must reconcile the inherent physical dimension variation present in fabrication of structures at these size scales [17], either through exacting fabrication tolerance and control or by design of electromagnetic solutions which are robust to such variation.

Acknowledgements. Supported by the Laboratory Directed Research and Development program at Sandia National Laboratories. Sandia National Laboratories is a multi-program laboratory managed and operated by Sandia Corporation, a wholly owned subsidiary of Lockheed Martin Corporation, for the U.S. Department of Energy's National Nuclear Security Administration under contract DE-AC04-94AL85000.

\section{References}

1. J.G. Fleming, S.Y. Lin, I. El-Kady, R. Biswas, K.M. Ho, Nature 417 (2002) 52.
2. C.M. Soukoulis, M. Wegener, Nat. Photon. 5 (2011) 523.

3. N. Liu, L. Fu, S. Kaiser, H. Schweizer, H. Giessen, Adv. Mater. 20 (2008) 4521.

4. J.K. Gansel, M. Thiel, M.S. Rill, M. Decker, K. Bade, V. Saile, G. von Freymann, S. Linden, M. Wegener, Science 325 (2009) 1513.

5. M.S. Rill, C. Plet, M. Thiel, I. Staude, G. von Freymann, S. Linden, M. Wegener, Nat. Mat. 7 (2008) 543.

6. J.H. Cho, D.H. Gracias, Nano Lett. 9 (2009) 4049.

7. J.H. Cho, M.D. Keung, N. Verellen, L. Lagae, V.V. Moshchalkov, P. Van Dorpe, D.H. Gracias, Small 7 (2011) 1943.

8. N. Liu, H. Guo, L. Fu, S. Kaiser, H. Schweizer, H. Giessen, Nat. Mat. 7 (2008) 31.

9. N. Liu, J. Liu, S. Zhu, H. Giessen, Stereometamaterials, Nat. Photon. 3 (2009) 157.

10. D.B. Burckel, C.M. Washburn, A.K. Raub, S.R.J. Brueck, D.R. Wheeler, S.M. Brozik, R. Polsky, Small 5 (2009) 2792.

11. S. Zhang, W. Fan, N.C. Panoiu, K.J. Malloy, R.M. Osgood, S.R.J. Brueck, Phys. Rev. Lett 95 (2005) 137404.

12. D.B. Burckel, J.R. Wendt, G.A. Ten Eyck, A.R. Ellis, I. Brener, M.B. Sinclair, Adv. Mater. 22 (2010) 3171.

13. D.B. Burckel, J.R. Wendt, G.A. Ten Eyck, J.C. Ginn, A.R. Ellis, I. Brener, M.B. Sinclair, Adv. Mater. 22 (2010) 5053.

14. D.B. Burckel, P.J. Resnick, P.S. Finnegan, M.B. Sinclair, P. Davids, Opt. Mater. Expr. 5 (2015) 2231.

15. N. Katsarakis, T. Koschny, M. Kafesaki, E.N. Economou, C.M. Soukoulis, Appl. Phys Lett. 84 (2004) 2943.

16. K. Aydin, I. Bulu, K. Guven, M. Kafesaki, C.M. Soukoulis, E. Ozbay, New J. Phys. 7 (2005) 168.

17. H. Gudjonson, M.A. Kats, K. Liu, Z. Nie, E. Kumacheva, F. Capasso, PNAS 111 (2014) E639.

Cite this article as: Adomanis BM, Resnick PJ \& Burckel DB: Reconciling measured scattering response of 3D metamaterials with simulation. EPJ Appl. Metamat. 2015, 2, 9. 